\title{
Proton-gated coincidence detection is a common feature of GPCR signaling
}

Nicholas J. Kapolka ${ }^{a}$, Jacob B. Rowe ${ }^{a}$, Geoffrey J. Taghon ${ }^{a}$, William M. Morgan ${ }^{a}$, Corin R. O'Shea ${ }^{a}$, and Daniel G. Isom ${ }^{a, b, c^{*}}$

From the aDepartment of Molecular and Cellular Pharmacology, University of Miami Miller School of Medicine, Miami, FL 33136; ' University of Miami Sylvester Comprehensive Cancer Center, Miami, FL 33136; and 'University of Miami Institute for Data Science and Computing, Miami, FL 33146

${ }^{*}$ Corresponding author:

Daniel G. Isom: disom@miami.edu

Key Words: GPCR, coincidence detection, agonism, antagonism, protons, proton sensors, logic gating, Boolean logic, acidosis 


\section{Abstract}

The evolutionary expansion of G protein-coupled receptors (GPCRs) has produced a rich diversity of transmembrane sensors for many physical and chemical signals. In humans alone, over 800 GPCRs detect stimuli such as light, hormones, and metabolites to guide cellular decision making primarily using intracellular $G$ protein signaling networks. This diversity is further enriched by GPCRs that function as molecular logic gates capable of discerning multiple inputs to transduce cues encoded in complex, context-dependent signals. Here, we show that many GPCRs are switch-like Boolean-gated coincidence detectors that link proton $\left(\mathrm{H}^{+}\right)$binding to GPCR signaling. Using a panel of 28 receptors, covering 280 individual GPCR-Ga coupling combinations, we show that $\mathrm{H}^{+}$gating both positively and negatively modulates and controls GPCR signaling. Notably, these observations extend to all modes of GPCR pharmacology including ligand efficacy, potency, and cooperativity. Additionally, we show that GPCR antagonism and constitutive activity are regulated by $\mathrm{H}^{+}$gating and report the discovery of a new acid sensor, the adenosine $\mathrm{A} 2 \mathrm{a}$ receptor (ADORA2A), which can be activated solely by acidic $\mathrm{pH}$. Together, these findings establish a new paradigm for GPCR biology and pharmacology in acidified microenvironments such as endosomes, synapses, tumors, and ischemic vasculature. 


\section{Introduction}

Over hundreds of millions of years, eukaryotes have evolved vast sensor arrays of G proteincoupled receptors (GPCRs) (1). The size and functionality of this receptor superfamily stems primarily from the structural adaptability of its 7-transmembrane (7TM) core. The versatility of the 7TM architecture has facilitated an extensive diversification and expansion of species-specific GPCR repertoires that can detect thousands of physical and chemical signals. In humans, more than 800 GPCRs detect inputs such as light, neurotransmitters, metabolites, and protons $\left(\mathrm{H}^{+}\right)$, the latter of which activate 3 receptors, GPR4, GPR65, and GPR68 in response to physiologic acidosis $(2,3)$. Outside of these three limited examples, the effect of $\mathrm{pH}$ on GPCR signaling and biology remains largely understudied.

Coincident signals are a well-recognized feature of neuronal and cellular communication $(4,5)$. By detecting simultaneous inputs, cells can filter signal from noise in complex chemical microenvironments to mount proper responses. As such, the combinatorial, spatial, and temporal nature of coincidence detection enables cells, tissues, and organs to integrate physiologic signals in a manner analogous to sensor array processing. At the molecular level, these activities are mediated by proteins such as NMDA receptor channels, which are synaptic coincidence detectors gated by glutamate and $\mathrm{Mg}^{2+}$ inputs that help choreograph the formation of long-term memories (5). Despite their abundance, similar roles for GPCRs as molecular coincidence detectors remain understudied and await exploration. Given that many GPCRs are already known to detect multiple orthosteric and allosteric inputs, there is likely an enormous potential for the discovery, understanding, and therapeutic utilization of molecular coincidence detection within the human GPCRome. 
Several lines of evidence suggest that coincidence detection is hard-wired into much of GPCR signaling biology. Recently, we have shown that acid sensing GPCRs GPR4, GPR65, and GPR68 are molecular coincidence detectors of $\mathrm{H}^{+}$and $\mathrm{Na}^{+}$ions. In this case, $\mathrm{H}^{+}$is an activator and $\mathrm{Na}^{+}$ is a negative allosteric modulator of GPR4, GPR65, and GPR68 signaling (6). Additionally, GPR68 has recently been shown to integrate $\mathrm{H}^{+}$and mechanical inputs in response to simultaneous extracellular acidification and membrane stretch (7). Other examples include the self-activation of the adhesion receptor, GPR56, that is mediated by coincident collagen-binding and shear force inputs (8), and input-specific synapse formation guided by the latrophilin-3 receptors and its coincident interactions with fibronectin leucine-rich repeat transmembrane proteins and teneurins (9). Here, we show that many other human GPCRs function as Booleangated coincidence detectors that couple $\mathrm{H}^{+}$binding to agonism, antagonism, and constitutive activity.

\section{Results and Discussion}

\section{Yeast are an ideal model system for $\mathrm{pH}$ studies of human GPCRs}

As transmembrane receptors, GPCRs can simultaneously experience different pH environments established across lipid bilayers. Localizing pH effects to the GPCR-ligand interface thus requires a model system with static intracellular $\mathrm{pH}\left(\mathrm{pH}_{\mathrm{i}}\right)$ independent of dynamic extracellular $\mathrm{pH}\left(\mathrm{pH}_{\mathrm{e}}\right)$. Such a model would avoid conflating $\mathrm{pH}_{\mathrm{e}}$ effects on GPCR signaling with changes in $\mathrm{pH}_{\mathrm{i}}$ or $\mathrm{pH}$ induced stress responses that affect processes such as intracellular signal transduction and cellular homeostasis. For example, we have previously shown that changes in $\mathrm{pH}_{\mathrm{i}}$ affect signaling by Ga subunits, the principal transducers of GPCR signals, and small Ras-like GTPases $(10,11)$. To circumvent such complications and ensure the interpretability of our results, we sought to identify a suitable cell model for studying $\mathrm{H}^{+}$gating of GPCR signaling. 
We began by testing the $\mathrm{pH}$ tolerance of human embryonic kidney cells (HEK293), the in vitro model most often used for cell-based GPCR assays. As shown in Fig. 1A, changes in $\mathrm{pH}_{\mathrm{e}}$ caused dramatic and unintended $\mathrm{pH}_{\mathrm{i}}$ alterations in HEK293 cells as reported by the $\mathrm{pH}$ biosensor pHluorin (12). This $\mathrm{pH}$ intolerance exhibited a sigmoidal shape centered at $\mathrm{pH} 6.25$ and asymptotic $\mathrm{pH}_{\mathrm{i}}$ values that differed by almost $1 \mathrm{pH}$ unit between high and low $\mathrm{pH}_{\mathrm{e}}$. In stark contrast, we found that $\mathrm{pH}_{\mathrm{i}}$ in $\mathrm{S}$. cerevisiae, a yeast cell model used for studying human GPCRs (13-15), was independent of $\mathrm{pH}_{\mathrm{e}}$ (Fig. 1B). Based on this breakthrough, we concluded that the cross-species compatibility and $\mathrm{pH}$ tolerance of yeast was ideal for $\mathrm{pH}$ studies of human GPCRs. Having already developed a high throughput platform for studying human GPCRs in yeast $(15,16)$, this new insight provided us with an experimental framework for modeling biological scenarios in which only the GPCR-ligand interface is exposed to large $\mathrm{pH}$ changes, such as those that occur in acidified endosomes and tumor microenvironments.

\section{pH profiling of human GPCRs using the yeast-based DCyFIR platform}

The fundamentals of GPCR signaling are conserved from yeast to humans. In mammalian cells, many GPCRs transduce signals through complex networks of intracellular signaling pathways. In contrast, yeast have only one GPCR pathway, controlled by the native yeast receptor Ste2, that is largely insulated from signal crosstalk $(17,18)$. Unlike human cells, which transduce most GPCR signals through $G_{\alpha}$ subunits, Ste2 signals through the $G_{\beta \gamma}$ heterodimer to activate a downstream MAP kinase cascade and transcriptional response. As illustrated in Fig. 1C, we have shown these processes are collectively insensitive to $\mathrm{pH}_{\mathrm{e}}$. As such, $\mathrm{pH}$ responses for human GPCRs in the yeast model cannot be attributed to $\mathrm{pH}$-induced changes in the underlying Ste2 pathway.

As shown in Fig. 1D, we have built a high throughput platform for studying human GPCRs that utilizes the isolated Ste2 pathway: Dynamic Cyan Induction by Functional Integrated Receptors, 
$\operatorname{DCyFIR}(6,15,16,19)$. In the DCyFIR model, activation of the yeast MAP kinase cascade is coupled to a human GPCR by a human/yeast C-terminal Ga chimera (Fig. 1D). To cover all possible human GPCR-Ga combinations, each GPCR is installed into a genome integrated cassette and expressed in 10 strains, each containing a mTurquoise2 (mTq2) transcriptional reporter and unique Ga chimera. Using this platform, we performed the foremost assessment of $\mathrm{H}^{+}$-gated GPCR activity by profiling the $\mathrm{pH}$ responses of 280 individual GPCR-Ga combinations, covering 28 unique receptors.

\section{Boolean $\mathrm{H}^{+}$gating of GPCR agonism}

As shown in Fig. 2A-B, we screened the set of 280 DCyFIR strains at $\mathrm{pH} 7$ and 5, identifying 131 GPCR-Ga agonist responses. 63 of these individual GPCR-Ga agonist responses exhibited signaling that was exclusive to $\mathrm{pH} 7$ or 5 . For example, the somatostatin receptor 5 (SSTR5) and all three sphingosine-1-phosphate receptors (S1PR1, S1PR2, and S1PR3) signaled only at pH 7. We characterized this all-or-nothing $\mathrm{H}^{+}$gating as switch-like Boolean behavior (Fig. 2A). In contrast, the remaining 68 individual GPCR-Ga agonist responses presented in Fig. 2B exhibited non-Boolean behavior by signaling to some degree at both $\mathrm{pH} 7$ and 5 . In some cases, such as FFAR2 and LPAR4, agonist responses were more challenging to quantify due to high levels of constitutive activity. In these cases, constitutive activity must be subtracted to observe the Boolean/non-Boolean agonist responses (see Supplemental Fig. S2B). Considering both Boolean and non-Boolean sets, we found that $66 \%$ of agonist responses were greater at $\mathrm{pH} 7$, $18 \%$ were greater at $\mathrm{pH} 5$, and $16 \%$ were similar at both $\mathrm{pH}$ values (Fig. $2 \mathrm{~A}-\mathrm{B}$ ). Having selected agonists with $\mathrm{pK}_{\mathrm{a}}$ values outside of $\mathrm{pH} 7$ and 5 (Supplemental Dataset 2), these effects were unlikely to be caused by changes in agonist ionization state. These findings show that for many GPCRs, signaling is more likely to be greater at higher $\mathrm{pH}$ and dramatically diminished or switched off at lower $\mathrm{pH}$ values. However, in rare cases we did find exceptions to this trend (FFAR2 and LPAR4 in Fig. 2A-B). 
An advantage of the yeast system is that it enabled us to measure the $\mathrm{pH}$ responses of individual GPCR-Ga interactions. In most cases, we observed that Ga coupling responses were similar for a given receptor and agonist. For example, all serotonin receptor 4 (HTR4) and lysophosphatidic acid receptor 4 (LPAR4) Ga pairs were more active at pH 7 and 5, respectively. However, Ga coupling patterns were unexpectedly complex in several cases, such as niacin and butyrate agonism of hydroxycarboxylic acid receptor 2 (HCAR2) (Fig. 2A-B and S2). Whereas $\mathrm{G}_{\mathrm{i} / \mathrm{o}}$ coupling was generally stronger at low $\mathrm{pH}$ for niacin, $\mathrm{G}_{\mathrm{q}}$ and $\mathrm{G}_{12 / 13}$ coupling favored high $\mathrm{pH}$. In contrast, butyrate agonism through $\mathrm{G}_{\mathrm{i} / \mathrm{o}}$ and $\mathrm{G}_{13}$ generally favored low $\mathrm{pH}$. These observations suggest that $\mathrm{H}^{+}$gating can be allosterically modulated by Ga coupling and that the net effect of $\mathrm{H}^{+}$gating on GPCR agonism could depend on the Ga repertoire expressed in a given cell type.

Our DCyFIR profiling experiments demonstrated that many GPCRs are Boolean and non-Boolean coincidence detectors of $\mathrm{H}^{+}$and agonists. As presented in Fig. 2A-C, this interpretation is consistent for both individual GPCR-Ga pairs and net Ga agonist responses for a given GPCR. As shown in Fig. 2C, the net $\mathrm{Ga}$ responses for $18 \mathrm{GPCR}$-agonist pairs exhibited Boolean behavior, with $17 / 1$ only signaling at $\mathrm{pH} 7 / 5$, respectively. A striking example of this switch-like $\mathrm{H}^{+}$-gated agonism is illustrated by titrations of dopamine receptor 4 (DRD4), for which signaling at $\mathrm{pH} 7$ was switched off at $\mathrm{pH} 6$ (Fig. 2D). Notably, of the 19 net Ga responses more active at $\mathrm{pH}$ 7, only 2 exhibited non-Boolean behavior (ADORA2B and LPAR3) (Fig. 2C). In contrast, of the 2 net Ga responses more active at pH 5 (LPAR4 and FFAR2), only LPAR4 exhibited switchlike Boolean signaling. The 9 remaining net $\mathrm{Ga}$ responses in Fig. $2 \mathrm{C}$ were insensitive to $\mathrm{pH}$, as illustrated in Fig. 2E by titrations of the melatonin receptor $1 \mathrm{~A}(\mathrm{MTNR} 1 \mathrm{~A})$ as a function of $\mathrm{pH}$. Together, these findings suggest that many GPCRs can respond to physiological pH changes like Boolean switches that are turned on and off by high and low $\mathrm{H}^{+}$concentrations. 


\section{All modes of GPCR pharmacology can be regulated by $\mathrm{H}^{+}$-gated coincidence detection}

As shown in Fig. 3, we next investigated the effects of $\mathrm{H}^{+}$gating on the major modes of GPCR pharmacology. Using a set of representative receptors and select Ga strains, we demonstrated that $\mathrm{H}^{+}$inputs can modulate agonist potency and efficacy (Fig. 3A-D), and receptor sensitivity and inhibition (Fig. 3E and 3F, respectively). As shown in Fig. 3A, the $\alpha_{2 B}$-adrenergic receptor (ADRA2B) appears to be insensitive to $\mathrm{pH}$ at high concentrations of the agonist epinephrine. However, $\mathrm{pH}$ has a profound effect on ADRA2B agonism at lower epinephrine concentrations, with increasingly lower $\mathrm{pH}$ values reducing epinephrine potency $\left(\mathrm{pEC}_{50}\right.$ values) by $>2$ orders of magnitude (Fig. 3A). A consequence of these $\mathrm{pH}$ dependent effects on epinephrine potency is that $\mathrm{H}^{+}$-gated agonism of ADRA2B exhibited near-Boolean behavior between $\mathrm{pH} 7$ and 5 , however only at concentrations between $\sim 10$ and $\sim 1000$ nanomolar. This finding illustrates that $\mathrm{H}^{+}$modulation of GPCR activity, or apparent lack thereof, can depend on agonist concentration.

In the case of ADRA2B, epinephrine elicits full agonist responses between $\mathrm{pH} 7$ and 5 (Fig. 3A). As such, the set of ADRA2B dose-response curves all achieved full terminal efficacy at high epinephrine concentration. In contrast, epinephrine agonism of the $\alpha_{2 A}$-adrenergic receptor (ADRA2A) was weaker and only reached full terminal efficacy at $\mathrm{pH} 7$ (Fig. 3B). At lower pH, ADRA2A achieved what we refer to as apparent terminal efficacy (at $\mathrm{pH} 6.5$ ) or did not signal. As a result, $\mathrm{H}^{+}$-gated ADRA2A agonism exhibited switch-like Boolean behavior between $\mathrm{pH} 7$ and 6 at micromolar epinephrine concentrations. The concept of terminal efficacy versus apparent terminal efficacy is further illustrated in Fig. 3C for serotonin receptor 4 (HTR4). In this case, serotonin agonism of HTR4 achieved terminal efficacy at $\mathrm{pH} 6.5$ and above, and apparent terminal efficacy at $\mathrm{pH} 6.0$ and below, leading to switch-like Boolean $\mathrm{H}^{+}$gating at micromolar serotonin concentrations. Lastly, as shown for hydroxycarboxylic acid receptor 2 (HCAR2), the effect of $\mathrm{pH}$ on agonism can be strictly limited to terminal efficacy (Fig. 3D). In this case, niacin 
agonism of HCAR2 maintained the same $\mathrm{pEC}_{50}$ value at all $\mathrm{pH}$ values yet displayed a dramatic increase in HCAR2 terminal efficacy with decreasing $\mathrm{pH}$.

As illustrated in Fig. 3E-F, $\mathrm{H}^{+}$-gated coincidence detection also extends to GPCR sensitization and inhibition. Fig. 3E shows how pH modulates cannabinoid receptor 2 (CNR2) responses to endogenous (2-arachidonoylglycerol, 2-AG) and synthetic (JWH-018 and HU-210) agonists. In the case of 2-AG, sensitivity of CNR2 signaling was non-cooperative and unaffected by $\mathrm{pH}$ as indicated by a near constant Hill coefficient $\left(n_{H}\right)$ between 0.9 to 1.5 . In contrast, $\mathrm{H}^{+}$gating of JWH018 and $\mathrm{HU}-210$ agonism elicited increasingly switch-like ultrasensitive responses $\left(n_{H}>2\right)(20)$ as $\mathrm{pH}$ was decreased. As shown in Fig. 3F, we observed a similar trend in $\mathrm{H}^{+}$gating of adenosine receptor A2A (ADORA2A) antagonism using the known competitive inhibitors caffeine, ZM241385, and $\mathrm{SCH}-58261$ in the presence of $10 \mu \mathrm{M}$ adenosine. All three inhibitors were $50 \%$ effective at $\mathrm{pH} 6$ and were almost completely ineffective at $\mathrm{pH} 5.0$. Based on these findings, we concluded that the potential for $\mathrm{H}^{+}$gating of GPCR pharmacology warrants far more attention in the development and evaluation of new and existing therapeutics. Our findings further suggest that as a principle of drug design, intentional $\mathrm{H}^{+}$gating may find broad utility in the development of drugs that target GPCRs exclusively in acidic microenvironments such as endosomes and tumors, and vice versa.

\section{New GPCR proton sensors}

As shown by the waterfall plots in Fig. 4A-B, 48 of the 280 individual GPCR-Ga strains screened at $\mathrm{pH} 7$ and 5 were constitutively active, with 24 responses showing switch-like Boolean behavior at $\mathrm{pH} 7$ or 5 (Fig. 4A). For example, the known acid sensor GPR68 signaled exclusively below pH 7. The 24 remaining GPCR-Ga responses in Fig. 4B exhibited non-Boolean behavior by signaling to some degree at both $\mathrm{pH} 7$ and 5. Considering both Boolean and non-Boolean sets, we found that $33 \%$ signaled higher at $\mathrm{pH} 7,54 \%$ signaled higher at $\mathrm{pH}$, and $13 \%$ were similar at both $\mathrm{pH}$ 
values (Fig. 4A-B). Unlike $\mathrm{H}^{+}$gating of GPCR agonism (Fig. 2), these findings show that GPCR constitutive activity is more likely to be greater at low $\mathrm{pH}$ in our receptor set. This result can be attributed to the high degree of Ga-coupling promiscuity we observed for known acid-sensing GPCRs (Fig. 4A-B, solid bars).

As shown in Fig. 4C, net Ga signaling for 7 of 12 constitutively active GPCRs exhibited Booleanlike behavior. Of these 7 receptors, 5 were activated by high $\mathrm{pH}$ (HCAR3, CNR2, ADRA2B, LPAR4, and HCAR2) and 2 by low pH (GPR68 and ADORA2A). Only 1 of the 12 receptors (FFAR2) exhibited non-Boolean behavior and higher signaling at $\mathrm{pH} 7$ (Fig. 4C). Notably, $\mathrm{H}^{+}$ gating of LPAR4 and FFAR2 constitutive activity (greater at $\mathrm{pH} 7$ ) was the inverse of their net $\mathrm{Ga}$ agonist responses (greater at $\mathrm{pH}$ ) shown in Fig. $2 \mathrm{C}$. This finding illuminates previously unknown complexities involving GPCR signaling and $\mathrm{pH}$ that likely extend to many other receptors. As shown by the $\mathrm{pH}$ titrations in Fig. 4D-E, we discovered a new acid sensor, ADORA2A (Fig. 4D), and a new class of $\mathrm{H}^{+}$sensors that are activated by high $\mathrm{pH}$ (Fig. 4E).

\section{The Boolean-like language of proton sensing and $\mathrm{H}^{+}$-gated GPCR coincidence detection}

Our findings demonstrate that proton sensing and $\mathrm{H}^{+}$-gated agonism are recurring features of GPCR signaling biology. Remarkably, the midpoint of proton-sensing and $\mathrm{H}^{+}$-gated responses $\left(\mathrm{pH}_{50}\right.$ values) are tightly grouped within the physiologic $\mathrm{pH}$ range, having an average value of 6.25 $\pm 0.75 \mathrm{pH}$ units (collectively for the $\mathrm{pH}$ titrations in Figs. 2-4). Focusing on the newly discovered acid sensor, ADORA2A, pH titrations through three GPCR-Ga pairs are in near-perfect agreement, all having $\mathrm{pH}_{50}$ values of $6.20 \pm 0.03$. This is similar to the $\mathrm{pH}_{50}$ of $6.35 \pm 0.02$ for the known acid sensor GPR68, which we have studied extensively (6). As shown in Fig. 4F, acidotic biological scenarios should activate ADORA2A in the absence of adenosine, such as situations in which ADORA2A is constitutively endocytosed (21). In contrast, our findings suggest that acidotic scenarios should negatively modulate most GPCR agonism (Fig. 2). For example, the 
switch-like Boolean inactivation of DRD4 between low and high $\mathrm{pH}$ could be a factor in the regulation of dopamine receptor signaling from endosomes $(22,23)$.

In summary, we have shown that much of GPCR proton sensing and $\mathrm{H}^{+}$-gated coincidence detection exhibits switch-like Boolean characteristics in the physiologic $\mathrm{pH}$ range. This concept is illustrated in Fig. 4G, in which we show that the effects of pH on many GPCRs can be described by a Boolean language expressed in concise binary codes. Moving forward, this formalism can be expanded to include a variety of additional inputs, such as allosteric modulators, to classify the complexities of GPCR logic gating. As such, we believe the technical and conceptual advances embodied in this work establish a new paradigm for understanding and exploring signaling in acidified microenvironments such as endosomes, tumors, synapses, and ischemic vasculature that will likely extend well beyond the GPCR superfamily. 


\section{Materials and Methods}

\section{Media}

All yeast strains were struck to YPD media plates $(20 \mathrm{~g} / \mathrm{L}$ peptone, $10 \mathrm{~g} / \mathrm{L}$ yeast extract, $2 \%$ glucose, $15 \mathrm{~g} / \mathrm{L}$ agar) from $30 \%$ glycerol stock cultures stored at $-80{ }^{\circ} \mathrm{C}$. For all DCyFIRscreen experiments and $\mathrm{pH}$ titrations, yeast were grown in low-fluorescence synthetic complete dropout media (SCD complete media; $50 \mathrm{mM}$ potassium phosphate dibasic, $50 \mathrm{mM}$ MES hydrate, $5 \mathrm{~g} / \mathrm{L}$ ammonium sulfate, $1.7 \mathrm{~g} / \mathrm{L}$ yeast nitrogen base without amino acids, folic acid, and riboflavin (Formedium; CYN6505), $0.79 \mathrm{~g} / \mathrm{L}$ complete amino acid mix (MP Biomedicals; 4500022), and 2\% glucose) titrated to the desired $\mathrm{pH}$ using $\mathrm{HCl}$ or $\mathrm{NaOH}$ and filter-sterilized. For yeast work using pHluorin to measure intracellular $\mathrm{pH}$, yeast were selected for on plates lacking leucine and grown in SCD media lacking leucine (5 g/L ammonium sulfate, $1.7 \mathrm{~g} / \mathrm{L}$ yeast nitrogen base without amino acids (MP Biomedicals; 4510522), $1 \mathrm{NaOH}$ pellet (VWR; BDH9292), 0.69 g/L CSM-LEU (amino acid mix lacking leucine), 2\% glucose, and $15 \mathrm{~g} / \mathrm{L}$ Bacto Agar for plates) that was filter-sterilized. For pHluorin purification experiments, autoinduction media was prepared by combining $800 \mathrm{~mL}$ ZY media (10 g/L tryptone, $5 \mathrm{~g} / \mathrm{L}$ yeast extract), $16 \mathrm{~mL}$ 50X 5052 (25\% (w/v) glycerol, 2.5\% (w/v) glucose, $10 \%$ (w/v) a-lactose), $16 \mathrm{~mL}$ 50X M (1.25 M Na $\mathrm{HPO}_{4}, 1.25 \mathrm{M} \mathrm{KH}_{2} \mathrm{PO}_{4}, 2.5 \mathrm{M} \mathrm{NH}_{4} \mathrm{Cl}$, $0.25 \mathrm{M} \mathrm{Na}_{2} \mathrm{SO}_{4}$ ), and $1.6 \mathrm{~mL} 1 \mathrm{M} \mathrm{MgSO}_{4}$. Phosphate buffered saline (PBS) was made by first preparing solutions of mono- and dibasic potassium phosphate (100 mM potassium chloride, 25 $\mathrm{mM}$ potassium phosphate). Individual $\mathrm{pH}$ solutions of PBS $(\mathrm{pH}=5.0-8.5$, increments of 0.25$)$ were prepared by mixing the mono- and dibasic solutions until the desired $\mathrm{pH}$ was reached, measured using an Accumet XL150 pH meter (Fisher Scientific; Hampton, NH). PBS-TCEP was prepared as PBS $(\mathrm{pH}=7.0)$ with $1 \mathrm{mM}$ TCEP, $0.15 \mathrm{mM}$ PMSF, $1 \mathrm{mM} \mathrm{MgCl}, 1 \mathrm{mM}$ EDTA, $0.1 \%$ Triton, spiked with lysozyme and $10 \mu \mathrm{L}$ DNAse. 


\section{Plasmids}

Yeast plasmid pYEplac181-pHluorin was a gift from the Rajini Rao lab at Johns Hopkins. The pHluorin gene from pYEplac181-pHluorin was subcloned into a pLIC-His vector (pLIC-HispHluorin) for recombinant overexpression and purification from E. coli. Mammalian plasmid pcDNA3.1(+)-pHluorin was made by synthesizing and cloning a codon optimized sequence of pHluorin into the pcDNA3.1(+) backbone (GenScript; Piscataway, NJ).

\section{Strains/Cell lines}

Yeast. Yeast strains used in this work were previously described in another study (15). Briefly, wild-type BY4741 was modified using CRISPR/Cas9 to prime the endogenous pheromone pathway (far1 $\Delta$, sst2 $\Delta$, ste2 $\Delta$ ) for expression of human GPCRs directly from the yeast genome (installation of an expression cassette $\left(P_{T E F}, T_{C Y C 1 B}\right)$ on chromosome $\mathrm{X}$ ), enable coupling of the yeast Ga to human GPCRs (via chimeric Ga proteins encoding the five C-terminal amino acids from human Ga proteins), and link receptor activation to a transcriptional reporter, mTurquoise2 (fig1 $1:: m$ Turquoise2). For a comprehensive list of all strains, see Dataset S1.

Mammalian. HEK293T (HEK293T/17) cells were purchased from American Type Culture Collections (ATCC; Gaithersburg, MD) and maintained in DMEM (Thermo Fisher; 11995-065) supplemented with 10\% Fetal Bovine Serum (Thermo Fisher; 10082-147) and 1\% PenicillinStreptomycin (Thermo Fisher; 15140-122).

\section{Intracellular pH measurements}

pHluorin purification. Ratiometric pHluorin (12) was overexpressed in E. coli using autoinduction. First, $25 \mathrm{ng}$ pLIC-His-pHluorin was transformed into competent BL21(DE3) RIPL cells. Transformants were grown overnight in $5 \mathrm{~mL}$ LB + carbenicillin $(100 \mu \mathrm{g} / \mathrm{mL}$; Sigma-Aldrich; C1389). The next morning, the overnight culture was transferred into $800 \mathrm{~mL}$ autoinduction media 
(see "Media" above), grown at $37^{\circ} \mathrm{C}$ with shaking (200 rpm) for 8 hours, then grown overnight at $18{ }^{\circ} \mathrm{C}$ with shaking (200 rpm). The $800 \mathrm{~mL}$ autoinduction culture was split over two $500 \mathrm{~mL}$ centrifuge bottles and cells were harvested by centrifugation at $4500 \mathrm{rpm}$ for 30 minutes. The supernatant was removed, and the pellet was resuspended in $35 \mathrm{~mL} P B S$, transferred to $50 \mathrm{~mL}$ tubes, and stored at $-20^{\circ} \mathrm{C}$. To purify overexpressed ratiometric $\mathrm{pHluorin}$, cell pellets were thawed on ice and treated with TCEP, $\mathrm{MgCl}_{2}$, EDTA (all to $1 \mathrm{mM}$ ), PMSF (to $0.15 \mathrm{mM}$ ), Triton (to $0.1 \%$ ), lysozyme, and $10 \mu \mathrm{L}$ DNAse, then lysed using a NanoDeBEE homogenizer (BEE International; South Easton, Massachusetts) at 30,000 psi. Cell lysate was transferred to a $50 \mathrm{~mL}$ tube and centrifuged at $15,000 \mathrm{rpm}$ for 30 minutes. Supernatant was then transferred to a $15 \mathrm{~mL}$ tube and incubated with $1 \mathrm{~mL}$ His bead slurry (Sigma-Aldrich; P6611) for 20 minutes at $4{ }^{\circ} \mathrm{C}$. Beads were harvested by centrifugation at $1000 \mathrm{rpm}$ for 1 minute, then washed five times with PBS-TCEP (see "Media" above). Beads were then transferred to a $2 \mathrm{~mL}$ spin column (Thermo Scientific; 89896) and eluted with PBS-TCEP + $200 \mathrm{mM}$ imidazole. Eluted protein was collected in a $15 \mathrm{~mL}$ tube by centrifugation at 1000 rpm for 1 minute, transferred to a slide-a-lyzer dialysis cassette (Thermo Scientific; 87730), and dialyzed overnight in 4 L PBS-TCEP at $4{ }^{\circ} \mathrm{C}$. Purified pHluorin stocks were stored at $-80^{\circ} \mathrm{C}$.

pHluorin calibration. A pHluorin standard curve was calculated using purified ratiometric pHluorin. Briefly, $2 \mu \mathrm{L}$ of ratiometric pHluorin $(100 \mu \mathrm{M})$ was resuspended in $198 \mu \mathrm{L}$ PBS buffer titrated to each pH (see "Media” above) and $50 \mu \mathrm{L}$ aliquots were moved into a 96-well microplate (CytoOne; CC7626-7596) in technical triplicate. Excitation spectra were collected using a ClarioStar microplate reader (BMG LabTech; Offenburg, Germany) with the following parameters: (top read, 40 flashes/well, excitation start: $340-10 \mathrm{~nm}$, excitation end: $495-10 \mathrm{~nm}, 1 \mathrm{~nm}$ steps; emission: 520-10 nm; instrument gain: 1700). Raw fluorescence values at 385 and $475 \mathrm{~nm}$ were used to calculate the pHluorin ratio $(385 / 475 \mathrm{~nm})$ for each replicate. A standard curve was built by plotting 
the pHluorin ratio as a function of $\mathrm{pH}$. Data was fit (sigmoidal, 4PL, $\mathrm{X}$ is $\log$ [concentration]) using GraphPad Prism (San Diego, California).

Measuring yeast intracellular $\mathrm{pH}$. Yeast intracellular $\mathrm{pH}$ measurements were collected using the $\mathrm{pH}$ biosensor ratiometric pHluorin expressed in the cytosol. $150 \mathrm{ng}$ of plasmid pYEplac181pHluorin was transformed into the wild-type yeast BY4741 using standard methods. Transformed cells were selected for on SD plates lacking leucine (see "Media" above). Eight individual colonies expressing cytosolic pHluorin were grown to mid-log phase in SCD media lacking leucine. Cells were harvested and washed three times in SCD Complete Media titrated to various $\mathrm{pH}$ values $(5.0,5.5,6.0,6.5,7.0,7.5,8.0)$, then transferred to a 384-well plate (Greiner; 781096). Spectral scans were collected using a ClarioStar microplate reader with the following parameters: top read, 40 flashes/well, excitation start: $340-10 \mathrm{~nm}$, excitation end: $495-10 \mathrm{~nm}, 5 \mathrm{~nm}$ steps; dichroic: 430 $\mathrm{nm}$; emission filter: 520-10 nm. pHluorin spectral scans were generated by subtracting the background autofluorescence of media at each $\mathrm{pH}$. pHluorin ratios were calculated from pHluorin spectral scans for each replicate by dividing the fluorescence at $385 \mathrm{~nm}$ by the fluorescence at $475 \mathrm{~nm}$. Intracellular $\mathrm{pH}$ was quantified using the pHluorin ratios as previously described (10).

Measuring mammalian cell intracellular $\mathrm{pH}$. Mammalian cell intracellular $\mathrm{pH}$ measurements were collected using the $\mathrm{pH}$ biosensor ratiometric pHluorin expressed in the cytosol. Prior to transfection, HEK293T cells were seeded into 6-well plates (CytoOne; CC7682-7506) at a density of 700,000 to 800,000 cells per well. Four hours later, cells were transfected with $2 \mu$ g plasmid DNA (pcDNA3.1(+) empty vector; $n=1$ well, or pcDNA3.1(+)-pHluorin; $n=8$ wells) complexed with $6 \mu \mathrm{L}$ TransIT-2020 transfection reagent (Mirus Bio; MIR 5404) at a final DNA concentration of 8 ng/uL in Advanced DMEM (Thermo Fisher; 12491-015). After 72 hours, cells were detached using TrypLE (Thermo Fisher; 12604-013), resuspended in $1 \mathrm{~mL}$ media (DMEM; 10\% FBS, 1\% PenStrep), and transferred to a $1.5 \mathrm{~mL}$ tube. Cells were harvested by centrifugation ( $600 \mathrm{rpm}, 3$ 
minutes) and resuspended in $600 \mu \mathrm{L}$ 1X HBSS (20 mM MES, $20 \mathrm{mM}$ HEPES). $100 \mu \mathrm{L}$ cell suspension was transferred to $61.5 \mathrm{~mL}$ tubes and harvested by centrifugation (600 rpm, 3 minutes). Cells were washed with 1X HBSS (20 mM MES, $20 \mathrm{mM}$ HEPES) titrated to each pH $(5.0,5.5,6.0,6.5,7.0$, and 7.5$)$ and transferred into a 384-well plate. A spectral scan of each well was collected using a ClarioStar microplate reader using the settings described above in "Measuring yeast intracellular $\mathrm{pH}$ ". pHluorin spectral scans were generated by subtracting the background autofluorescence of cells transfected with pcDNA3.1(+) from those transfected with pcDNA3.1(+)-pHluorin. pHluorin ratios were calculated from pHluorin spectral scans for each replicate by dividing the fluorescence at $385 \mathrm{~nm}$ by the fluorescence at $475 \mathrm{~nm}$. Intracellular $\mathrm{pH}$ was quantified using the pHluorin ratios as previously described (10).

\section{DCyFIRscreen profiling of GPCR proton-linked coincidence detection}

For all DCyFIRscreen experiments in this study, individual strains were grown to mid-log phase in SCD complete media titrated to either $\mathrm{pH} 5.0$ or 7.0. Cells were harvested and normalized to a starting $\mathrm{OD}_{600}=0.1$ before treatment with vehicle or agonist in both $\mathrm{pH}$ medias. Cells were incubated at $30{ }^{\circ} \mathrm{C}$ until cell densities between the two $\mathrm{pH}$ medias were similar $\left(\mathrm{OD}_{600} \sim 3-4 ; \sim 18\right.$ hours at $\mathrm{pH} 5.0, \sim 24-48$ hours at $\mathrm{pH} 7.0$ ) before $\mathrm{mTq} 2$ fluorescence was measured using a ClarioStar microplate reader (bottom read, 10 flashes/well, excitation filter: $430-10 \mathrm{~nm}$; dichroic filter: LP $458 \mathrm{~nm}$; emission filter: 482-16 nm; gain = 1,300). mTq2 fluorescence for treated/untreated cells at pH 5.0 and 7.0 was plotted using GraphPad Prism. For plots displaying DCyFIRscreen data "relative to basal", mTq2 fluorescence from untreated cells was used as baseline and subtracted from the $\mathrm{mTq} 2$ fluorescence from treated cells. 


\section{Analysis of raw DCyFIRscreen data}

Agonist response. In total, 340 DCyFIRscreen experiments covering 28 unique receptors (280 GPCR-Ga strains) treated with vehicle or agonist(s) were analyzed. A given GPCR-Ga strain was considered responsive to agonist if treated cells exhibited a minimal $\mathrm{mTq} 2$ fluorescence $\geq 25,000$ RFU and if the $\log _{2}$ fold-change $\left(\log _{2} F C\right)$ of $\mathrm{mTq} 2 \mathrm{RFU}$ from treated relative to untreated cells was $\geq 0.5$ at either $\mathrm{pH} 5.0$ or 7.0 . In one case (ADORA2A $\mathrm{G}_{\alpha, 12}$ ), we observed more mTq2 fluorescence from untreated cells than treated cells. Although this strain met the requirements described above, the ligands used in the screen were strictly agonists. As such, this strain was removed from the set of agonist-responsive GPCR-Ga strains. We also excluded another strain (ADRA2B $G_{\alpha, 13}$ treated with epinephrine) from the set of agonist-responsive GPCR-Ga strains after it was experimentally determined to be a false-positive (data not shown). Using these criteria, we identified 131 agonist-responsive GPCR-Ga strains and calculated their raw agonist response (treated mTq2 RFU - untreated mTq2 RFU) for each replicate at pH 7.0 and 5.0.

Agonist response, Boolean criteria. An agonist-responsive GPCR-Ga strain was considered to exhibit Boolean-like behavior if the mean raw agonist response at one $\mathrm{pH}$ was near zero (e.g. less than the mean of the corresponding vehicle-treated controls), suggesting all-or-nothing agonism between both $\mathrm{pH}$ values. In a small subset of cases where agonist response was weak and appeared to be Boolean (ADORA1 $\mathrm{G}_{\alpha, 15}+$ adenosine, CNR2 $\mathrm{G}_{\alpha, 12}+$ HU-210, PTGER3 $\mathrm{G}_{\alpha, 13}$ + prostaglandin E2, LPAR3 $\mathrm{G}_{\alpha, 12}+\mathrm{LPA}$, and FFAR2 $\mathrm{G}_{\alpha, 15}+$ acetate), we could not rule out that a lack of signaling at either $\mathrm{pH}$ was due to weak (e.g. all-or-nothing) signaling. In these cases, and consistent with our conservative approach to classifying Boolean-like behavior, we labeled these examples as non-Boolean. Using these criteria, we identified 63 agonist-responsive GPCR-Ga strains that exhibited Boolean-like behavior, and 68 that exhibited non-Boolean-like behavior. Differential agonism was then determined by calculating the $\log _{2} \mathrm{FC}$ of $\mathrm{mTq} 2 \mathrm{RFU}$ from treated cells relative to untreated cells at active $\mathrm{pH}$ (for Boolean-like responses) or raw agonist responses 
between cells treated at $\mathrm{pH} 7.0$ relative to $\mathrm{pH} 5.0$ (for non-Boolean-like responses) for all replicates.

Net $\mathrm{G} \alpha$ agonist response. For a given receptor-agonist pair, net $\mathrm{G} \alpha$ agonist response was calculated as the sum of mTq2 RFU from treated and untreated agonist-responsive GPCR-Ga strains for each replicate at $\mathrm{pH} 5.0$ and 7.0. The raw net $\mathrm{G} \alpha$ agonist response was determined by subtracting the net $\mathrm{Ga}$ agonist response of untreated cells from treated cells at pH 5.0 and 7.0 for each replicate. A net $\mathrm{G} \alpha$ agonist response was considered to exhibit Boolean-like behavior if the mean raw net $\mathrm{Ga}$ agonist response at one $\mathrm{pH}$ was near zero (e.g. less than the sum of the corresponding untreated controls). Using these criteria, we identified 18 net Ga agonist responses that exhibited Boolean-like behavior and 12 that exhibited non-Boolean-like behavior. Differential agonism of net $\mathrm{G} \alpha$ agonist response was determined by calculating the $\log _{2} \mathrm{FC}$ of the net $\mathrm{G} \alpha$ agonist response from treated cells relative to untreated cells at active $\mathrm{pH}$ (for Boolean-like responses) or from the raw net $\mathrm{G} \alpha$ agonist response between cells treated at $\mathrm{pH} 7.0$ and $\mathrm{pH} 5.0$ (for non-Boolean-like responses) for all replicates.

Constitutive activity. In total, 28 unique receptors (280 GPCR-Ga strains) were assessed for constitutive activity. A given GPCR-Ga strain was considered constitutively active if the $\log _{2}$ FC of the mean of untreated cells relative to untreated controls was $\geq 1.0$. In one case (ADORA1 $G_{\alpha, t}$ ), we excluded a GPCR-Ga strain from the set of constitutively active strains after it was experimentally determined to be a false positive (data not shown). This selection criteria identified 48 unique constitutively active GPCR-Ga strains.

Constitutive activity, Boolean criteria. A constitutively active GPCR-Ga strain was considered to exhibit Boolean-like behavior if the mean mTq2 RFU for untreated cells at one $\mathrm{pH}$ was near zero (e.g. less than twice the mTq2 RFU of untreated controls). Using these criteria, we identified 24 
GPCR-G $\alpha$ strains that exhibit Boolean-like constitutive activity and 24 that exhibited non-Booleanlike constitutive activity. Differential constitutive activity was quantified by calculating the $\log _{2} \mathrm{FC}$ of the mTq2 RFU between untreated cells at $\mathrm{pH} 7.0$ and 5.0 for each replicate.

Net Ga constitutive activity. Net Ga constitutive activity was determined by calculating the sum of mTq2 RFU for all constitutively active GPCR-Ga strains for a given receptor at $\mathrm{pH} 7.0$ and 5.0 for each replicate. The constitutive activity of a given GPCR was considered to exhibit Boolean-like behavior if the mean net Ga constitutive activity at one $\mathrm{pH}$ was near zero (e.g. less than twice the mean mTq2 RFU of the corresponding untreated controls). Using these criteria, we identified 7 receptors that exhibited Boolean-like net $\mathrm{Ga}$ constitutive activity and 3 that exhibited nonBoolean-like constitutive activity. In the specific case of ADORA2A, 5 of the 6 constitutively active GPCR-Ga strains exhibited Boolean-like responses. Based on this finding, and on our conservative approach for characterizing the effects of $\mathrm{pH}$ on receptor behaviors, we classified ADORA2A as Boolean. Differential net Ga constitutive activity was determined by calculating the $\log _{2} \mathrm{FC}$ of the sum of untreated constitutively active GPCR-Ga strains at $\mathrm{pH} 7.0$ relative to 5.0 for each replicate.

\section{Ligand and constitutive activity titrations}

Ligand titrations as a function of $\mathrm{pH}$. Individual strains were grown to mid-log phase in SCD complete media titrated to each $\mathrm{pH}(5.0,5.5,6.0,6.5$, and 7.0$)$. Cells were harvested and normalized to a starting $\mathrm{OD}_{600}=0.1$ in each $\mathrm{pH}$ media. $36 \mu \mathrm{L}$ normalized cells at each $\mathrm{pH}$ were moved to a 384-well microplate with $4 \mu \mathrm{L} 10 \mathrm{X}$ ligand stocks in a 16-point serial dilution series centered around the apparent $\mathrm{pEC}_{50}$ at $\mathrm{pH}=7.0$. Plates were sealed with a permeable lid (Diversified Biotech; BERM-2000) and incubated at $30{ }^{\circ} \mathrm{C}$. At least seven fluorescence and absorbance measurements were recorded in 1-3 hr intervals over $\sim 24$ hours. Fluorescence values were plotted as a function of absorbance for each ligand concentration at each $\mathrm{pH}$ and fit 
(simple linear regression) using GraphPad Prism. Slope values from the fit function were then plotted for each ligand concentration at each $\mathrm{pH}$. At least two points from the relative baseline of each curve were averaged and used as baseline for background correction (e.g. "relative to basal"). Data were fit (log(agonist) vs. response -- Variable slope (four parameters)) using GraphPad Prism. In cases where data was normalized from $0-100 \%$, constraints of bottom $=0$ and top $=100$ were used in the fit function.

Constitutive activity titrations as a function of $\mathrm{pH}$. Individual strains were grown to mid-log phase in SCD complete media titrated to $\mathrm{pH} 7.0$ (for strains with higher constitutive activity at low $\mathrm{pH}$ ) or to $\mathrm{pH} 5.0$ (for strains with higher constitutive activity at high $\mathrm{pH}$ ). Cells were harvested and normalized to a starting $\mathrm{OD}_{600}=0.5$ then washed twice in SCD complete media titrated to each $\mathrm{pH}(5.0,5.5,5.75,6.0,6.25,6.5,6.75,7.0,7.25$, and 7.5$) .40 \mu \mathrm{L}$ cells were moved into a 384-well microplate, sealed with a permeable lid, and incubated at $30^{\circ} \mathrm{C}$. Fluorescence and absorbance measurements were collected, and data was analyzed as described above in "Ligand titrations as a function of $\mathrm{pH}^{\prime \prime}$.

\section{Confocal Microscopy}

Yeast. Wild-type yeast transformed with pYEplac181-pHluorin (see "Measuring yeast intracellular pH" above for details) were grown to mid-log phase in media lacking leucine. Cells were normalized to an $\mathrm{OD}_{600}=1.0$ in low fluorescence media (see "Media" above), transferred to an $\sim 1 \mathrm{~cm}$ square agar "pad" on a glass microscope slide (VWR; 16004-422) and covered with a glass coverslip (VWR; 48366-227). Fluorescence images were collected using an LSM800 confocal microscope (Zeiss; Oberkochen, Germany) with the following parameters: pinhole 1.00 AU/44 $\mu \mathrm{m}$; laser wavelength $488 \mathrm{~nm}, 1.0 \%$ intensity; $495 \mathrm{~nm}$ excitation; $520 \mathrm{~nm}$ emission. 
HEK293T. Cells transfected with pcDNA3.1(+)-pHluorin (see "Measuring mammalian intracellular $p H^{\prime \prime}$ above for details) were detached with TrypLE (Thermo Fisher; 12604-013) and transferred to confocal dishes (VWR; 75856-742) at a density of 300,000 cells/dish in a total of $3 \mathrm{~mL}$ media (DMEM; 10\% FBS, 1\% PenStrep). After 48 hours, fluorescence images were collected using an LSM800 confocal microscope (Zeiss; Oberkochen, Germany) with the following parameters: pinhole $0.67 \mathrm{AU} / 32 \mu \mathrm{m}$; laser wavelength $488 \mathrm{~nm}, 3.0 \%$ intensity; $495 \mathrm{~nm}$ excitation; $520 \mathrm{~nm}$ emission.

\section{Acknowledgements}

This work was supported by the National Institutes of Health through the National Institute of General Medical Sciences (R35GM119518) to D.G.I.

Author Contributions: D.G.I. and N.J.K. wrote the manuscript. N.J.K. engineered yeast strains and performed the experiments. J.B.R., G.J.T., and C.R.O. engineered yeast strains. W.M.M. provided technical support. J.B.R. and G.J.T. reviewed and revised the manuscript.

Competing Interest Statement: The authors declare no competing interests.

Data availability. All relevant data, protocols, results, and analyses are available in the main text. All computer code and yeast strains associated with this work are available upon request. 


\section{References}

1. R. Strotmann et al., Evolution of GPCR: change and continuity. Mol Cell Endocrinol 331, 170-178 (2011).

2. M. G. Ludwig et al., Proton-sensing G-protein-coupled receptors. Nature 425, 93-98 (2003).

3. J. Q. Wang et al., TDAG8 is a proton-sensing and psychosine-sensitive G-proteincoupled receptor. J Biol Chem 279, 45626-45633 (2004).

4. J. G. Carlton, P. J. Cullen, Coincidence detection in phosphoinositide signaling. Trends Cell Biol 15, 540-547 (2005).

5. T. Miyashita et al., $\mathrm{Mg}(2+)$ block of Drosophila NMDA receptors is required for long-term memory formation and CREB-dependent gene expression. Neuron 74, 887-898 (2012).

6. J. B. Rowe et al., The molecular evolution and mechanism of $\mathrm{G}$ protein-coupled receptor proton sensing. J Biol Chem (2020).

7. W. C. Wei et al., Coincidence Detection of Membrane Stretch and Extracellular pH by the Proton-Sensing Receptor OGR1 (GPR68). Curr Biol 28, 3815-3823 e3814 (2018).

8. J. Yeung et al., GPR56/ADGRG1 is a platelet collagen-responsive GPCR and hemostatic sensor of shear force. Proc Natl Acad Sci U S A 117, 28275-28286 (2020).

9. R. Sando, X. Jiang, T. C. Sudhof, Latrophilin GPCRs direct synapse specificity by coincident binding of FLRTs and teneurins. Science 363 (2019).

10. D. G. Isom et al., Protons as second messenger regulators of $\mathrm{G}$ protein signaling. Mol Cell 51, 531-538 (2013).

11. D. G. Isom, V. Sridharan, H. G. Dohlman, Regulation of Ras Paralog Thermostability by Networks of Buried lonizable Groups. Biochemistry 55, 534-542 (2016).

12. G. Miesenbock, D. A. De Angelis, J. E. Rothman, Visualizing secretion and synaptic transmission with pH-sensitive green fluorescent proteins. Nature 394, 192-195 (1998).

13. S. J. Dowell, A. J. Brown, Yeast assays for G-protein-coupled receptors. Recept Channels 8, 343-352 (2002).

14. S. J. Dowell, A. J. Brown, Yeast assays for G protein-coupled receptors. Methods Mol Biol 552, 213-229 (2009).

15. N. J. Kapolka et al., DCyFIR: a high-throughput CRISPR platform for multiplexed G protein-coupled receptor profiling and ligand discovery. Proc Natl Acad Sci U S A 117, 13117-13126 (2020).

16. J. B. Rowe, G. J. Taghon, N. J. Kapolka, W. M. Morgan, D. G. Isom, CRISPRaddressable yeast strains with applications in human $\mathrm{G}$ protein-coupled receptor profiling and synthetic biology. J Biol Chem 295, 8262-8271 (2020). 
17. L. Bardwell, A walk-through of the yeast mating pheromone response pathway. Peptides 26, 339-350 (2005).

18. Y. Wang, H. G. Dohlman, Pheromone signaling mechanisms in yeast: a prototypical sex machine. Science 306, 1508-1509 (2004).

19. N. J. Kapolka, D. G. Isom, HCAR3: an underexplored metabolite sensor. Nat Rev Drug Discov 19, 745 (2020).

20. C. Y. Huang, J. E. Ferrell, Jr., Ultrasensitivity in the mitogen-activated protein kinase cascade. Proc Natl Acad Sci U S A 93, 10078-10083 (1996).

21. J. Lu et al., An Anti-Parkinson's Disease Drug via Targeting Adenosine A2A Receptor Enhances Amyloid-beta Generation and gamma-Secretase Activity. PLoS One 11, e0166415 (2016).

22. S. J. Kotowski, F. W. Hopf, T. Seif, A. Bonci, M. von Zastrow, Endocytosis promotes rapid dopaminergic signaling. Neuron 71, 278-290 (2011).

23. Y. Li et al., Identification of two functionally distinct endosomal recycling pathways for dopamine D(2) receptor. J Neurosci 32, 7178-7190 (2012). 


\section{Figures}

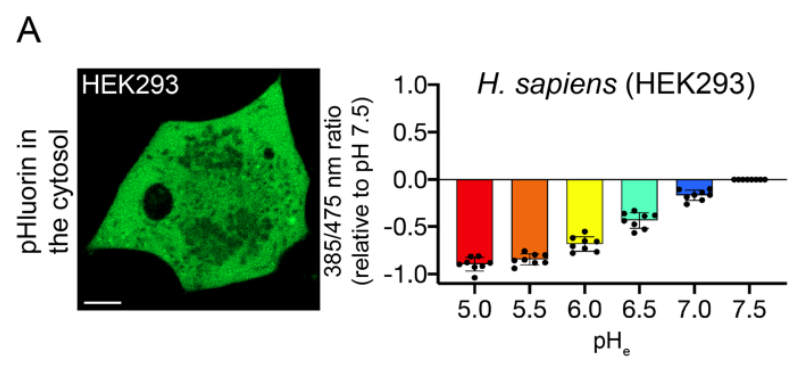

B

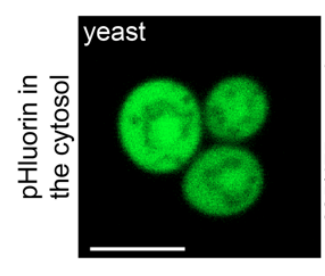

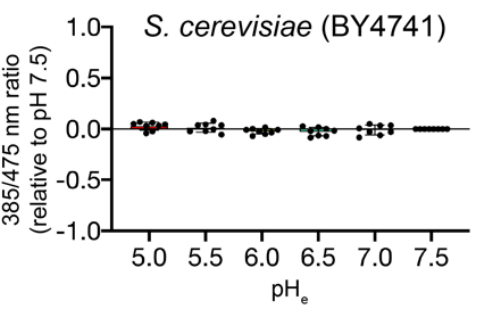

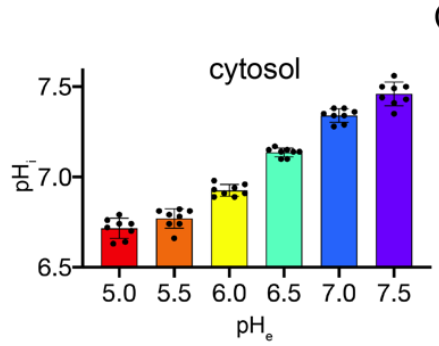

C

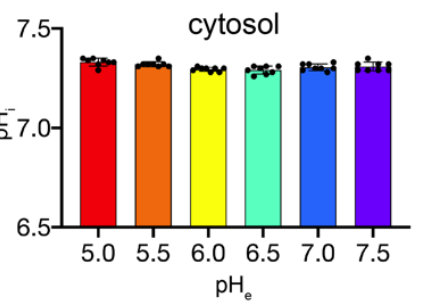

Ste2

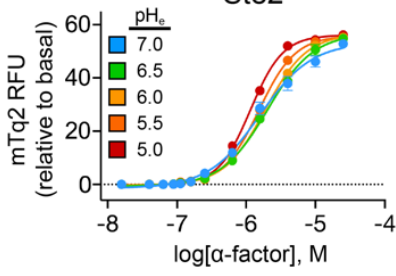

D

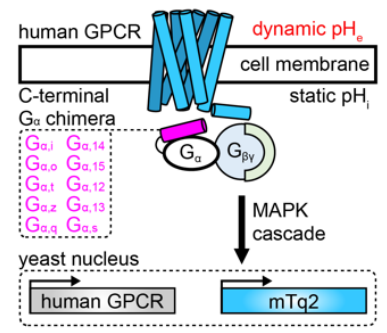

Fig. 1. Using yeast to study $\mathrm{H}^{+}$-gated coincidence detection by human GPCRs. Confocal images, wavelength ratios, and $\mathrm{pH}_{\mathrm{i}}$ values reported by the $\mathrm{pH}$ biosensor pHluorin in human embryonic kidney (HEK293) (A) and yeast cells (S. cerevisiae strain BY4741) (B). Error bars represent $\mathrm{SD}$ of $\mathrm{n}=8$ biological replicates. Scale bars, $5 \mu \mathrm{m}$. Calibration experiments are provided in Fig. S1. (C) The native yeast GPCR, Ste2, and its downstream pathway components are not affected by changes in $\mathrm{pH}_{\mathrm{e}}$. Error bars represent $\mathrm{SD}$ of $\mathrm{n}=4$ experimental replicates. (D) In the DCyFIR platform, human GPCRs are integrated into a CRISPR-addressable expression cassette in 10 DCyFIR strains, each of which contains a single, unique C-terminal Ga chimera (pink) to test all possible GPCR-Ga coupling combinations. Receptor activation drives the expression of the $\mathrm{mTq} 2$ transcriptional reporter protein. 


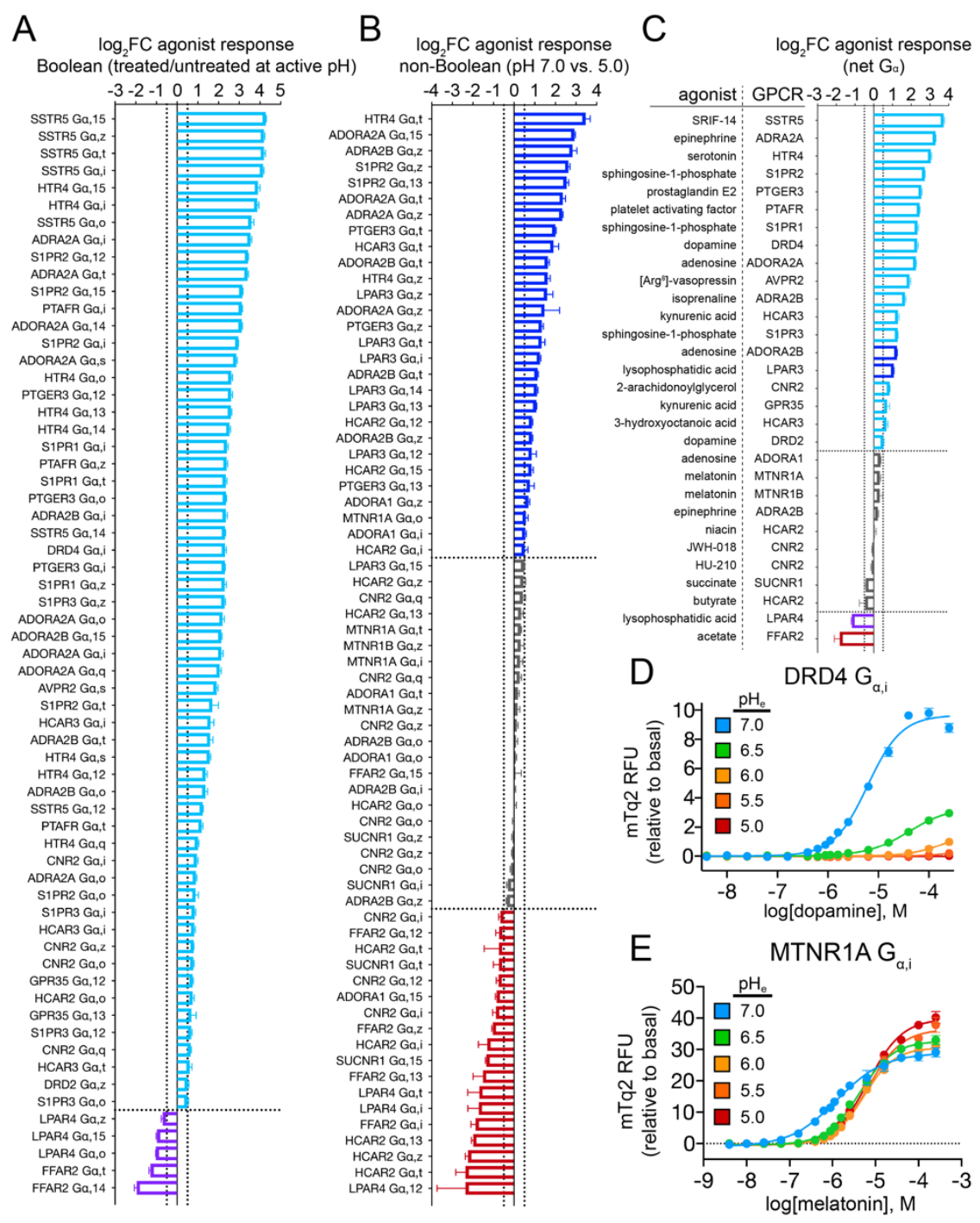

Fig. 2. $\mathrm{H}^{+}$-gated coincidence detection for $131 \mathrm{GPCR}-\mathrm{G} \alpha$ coupling combinations. Waterfall plots showing Boolean $(A)$ and non-Boolean $(B) \mathrm{H}^{+}$-gated agonist responses. Differential agonism was quantified as the $\log _{2}$ fold change $\left(\log _{2} \mathrm{FC}\right)$ of the ratio of agonist treated and untreated samples at active $\mathrm{pH}(\mathrm{A})$ or agonist response between $\mathrm{pH} 7$ and 5 (B). (C) Waterfall plot showing the net Ga agonist responses for a given receptor using the ratio of summed activity for all agonist-active GPCR-Ga strains between $\mathrm{pH} 7$ and 5. Switch-like Boolean responders to high and low $\mathrm{pH}$ are colored cyan and purple, respectively. (D) Switch-like Boolean behavior exhibited by dopamine receptor 4 (DRD4 $\mathrm{G}_{\alpha, \mathrm{i}}$ ). (E) pH-insensitive GPCR signaling by melatonin receptor $1 \mathrm{~A}\left(\mathrm{MTNR} 1 \mathrm{~A} \mathrm{G}_{\alpha, \mathrm{i}}\right)$. (A-C) Vertical dashed lines correspond to a $\log _{2} \mathrm{FC}=0.5$ and 
horizontal dashed lines separate examples that signaled more $\left(\log _{2} \mathrm{FC} \geq 0.5\right)$ at $\mathrm{pH} 7$ (blue/cyan bars), similarly ( $\log _{2} \mathrm{FC}$ between \pm 0.5 ) between both $\mathrm{pH}$ values (gray bars), or more $\left(\log _{2} \mathrm{FC} \leq\right.$ 0.5) at $\mathrm{pH} 5$ (red/purple bars). (A, B) Primary screening data is provided in Fig. S2. (A-E) Error bars represent SD of $n=4$ experimental replicates. 
A

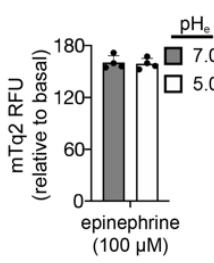

B

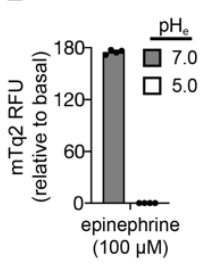

E
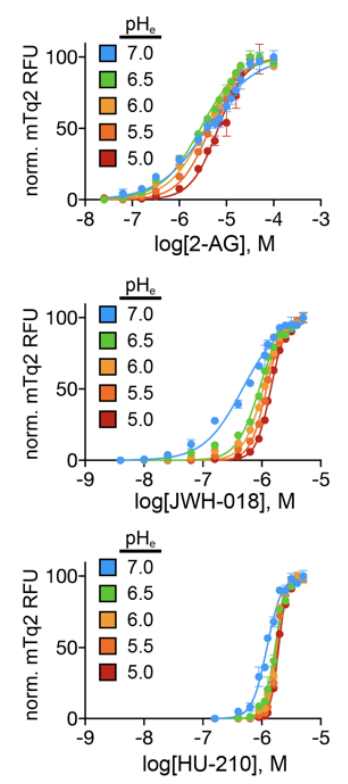

ADRA2B $G_{\alpha, i}$

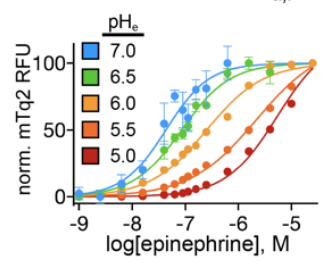

ADRA2A $G_{a, i}$
C

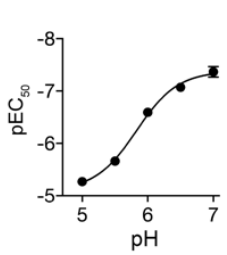

$\mathrm{pH}$

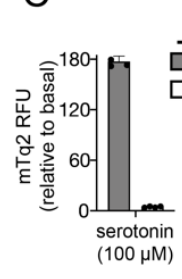

D
HTR4 $\mathrm{G}_{\alpha, \mathrm{i}}$

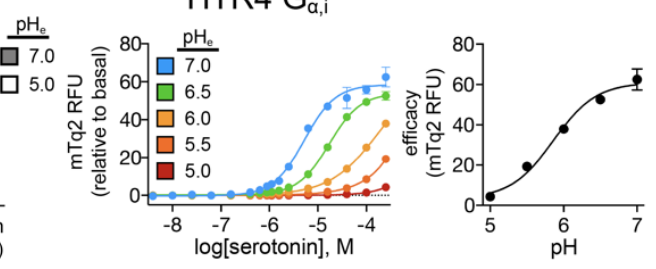

HCAR2 $G_{\alpha, t}$
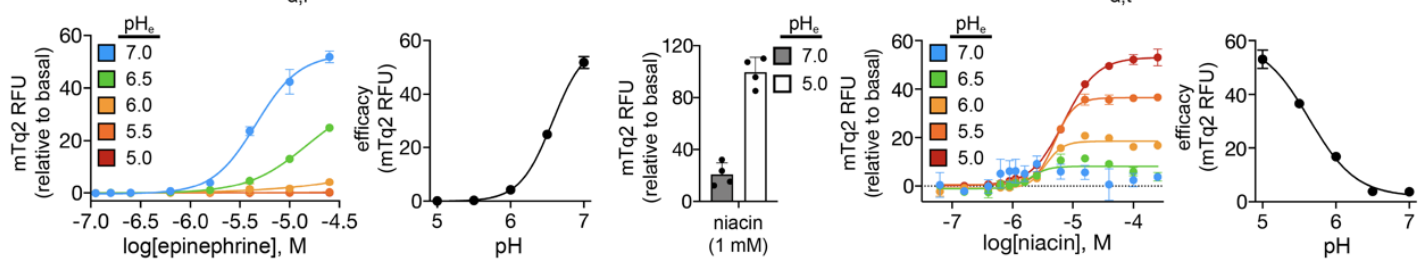

F

ADORA2A $G_{a, 0}$
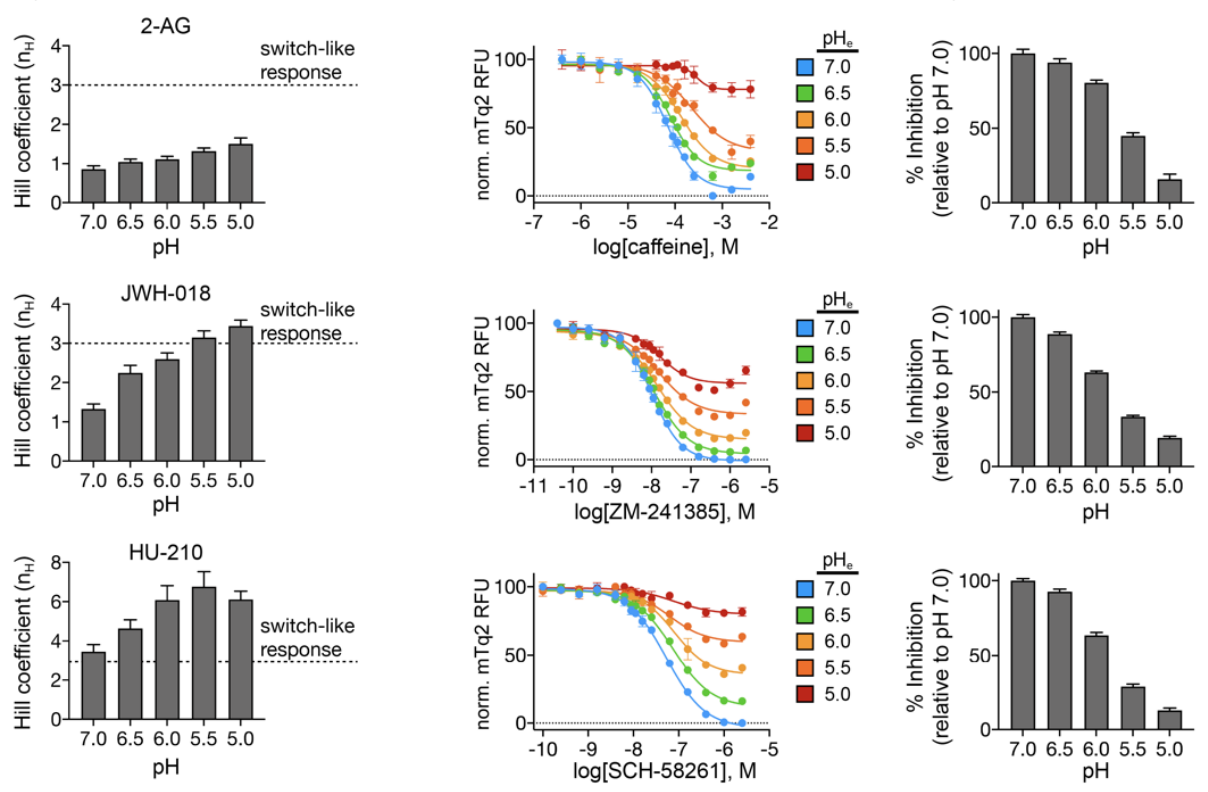

Fig. 3. Pharmacological modalities of $\mathbf{H}^{+}$-gated coincidence detection by GPCRs.

(A) Potentiation of agonist potency. Endpoint measurements of ADRA2B agonism (left) at pH 7 (gray bars) and 5 (white bars). Epinephrine dose-response curves (middle) and $\mathrm{pEC}_{50}$ values (right) for ADRA2B. (B-D) Potentiation of agonist efficacy. Endpoint measurements (left) of ADRA2A (B), HTR4 (C), and HCAR2 (D) agonism at pH 7 (gray bars) and 5 (white bars). Doseresponse curves (middle) and efficacy (right) of each GPCR with its endogenous agonist. (E) Potentiation of sensitivity. 2-arachidonoyl glycerol (2-AG) (top), JWH-018 (middle), and HU210 (bottom) dose-response curves (left) and Hill coefficients (right) for CNR2. (F) Potentiation of 
bioRxiv preprint doi: https://doi.org/10.1101/2021.01.08.425945; this version posted January 9, 2021. The copyright holder for this preprint

(which was not certified by peer review) is the author/funder, who has granted bioRxiv a license to display the preprint in perpetuity. It is made available under aCC-BY 4.0 International license.

inhibition/antagonism. Caffeine (top), ZM-241385 (middle), and SCH-58261 (bottom) doseresponse curves (left) and percent inhibition (right) for ADORA2A in the presence of $10 \mu \mathrm{M}$ adenosine. (A-F) Error bars represent SD of $n=4$ experimental replicates. 


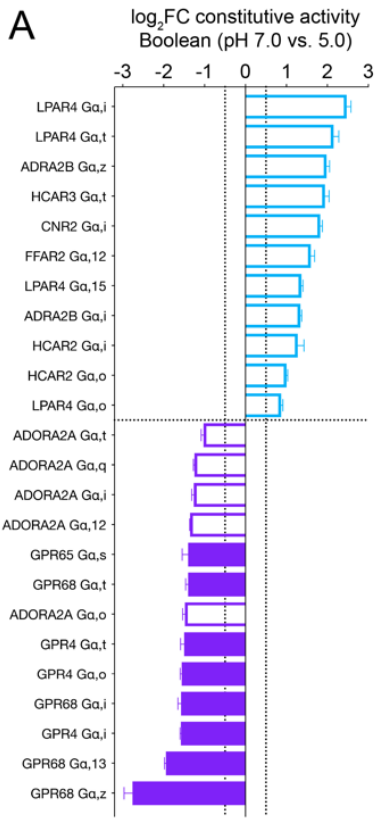

$\mathrm{F}$

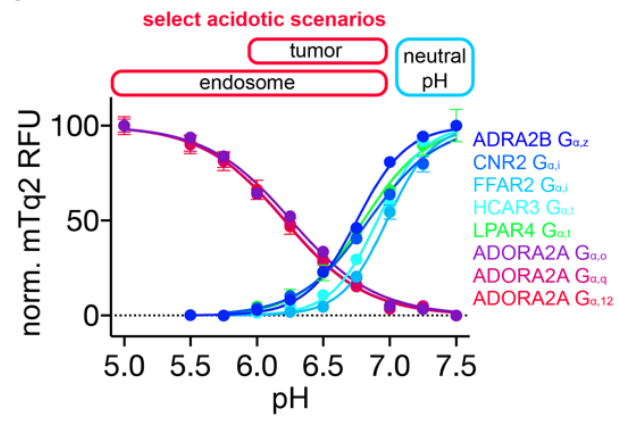

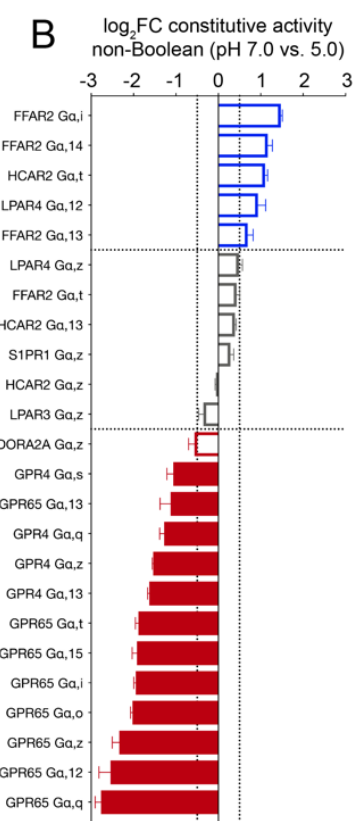
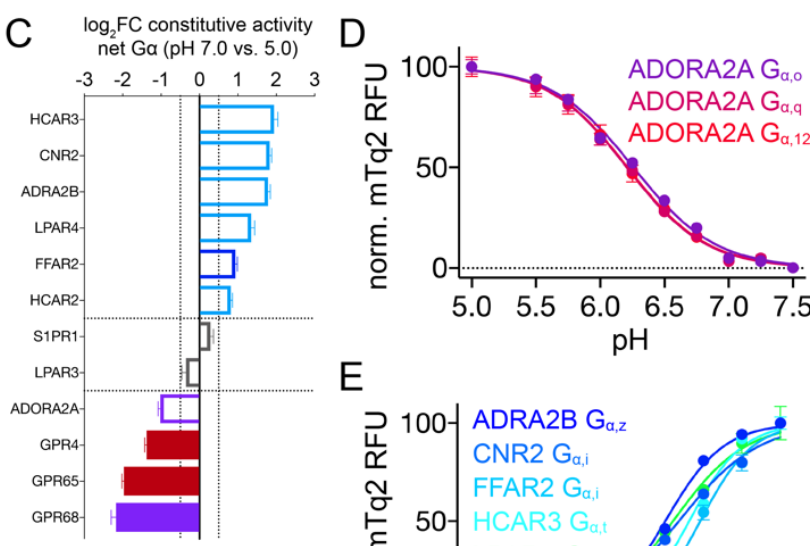

E

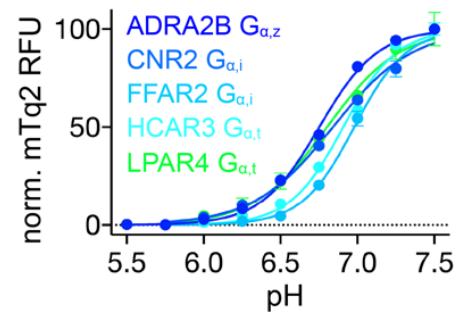

G

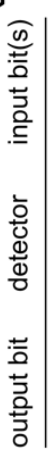

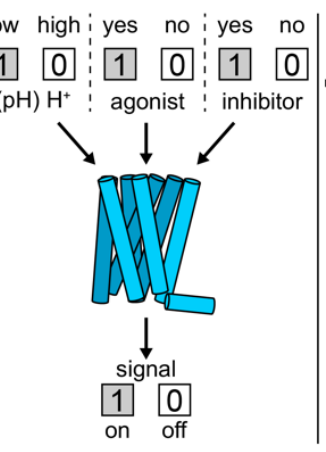

$0,10: 1$.

binary code

proton sensing example

0 0 $0: 0$ ADORA2A at $\mathrm{pH} 7$

$100: 1$ ADORA2A at $\mathrm{pH} 5$

$\mathrm{H}^{+}$-gated agonism

$0,10: 1$ DRD4 + dopamine at $\mathrm{pH} 7$
$110: 0$ DRD4 + dopamine at pH 5

Fig. 4. New pH sensors and Boolean logic of $\mathrm{H}^{+}$-gated GPCR signaling. (A, B) Waterfall plots showing Boolean (A) and non-Boolean (B) instances of $\mathrm{H}^{+}$-gated GPCR-G $\alpha$ constitutive activity. Differential signaling was quantified as the $\log _{2} \mathrm{FC}$ between $\mathrm{pH} 7$ and 5 . (C) Waterfall plot showing the net $\mathrm{G} \alpha$ responses for a given receptor using the ratio of summed mTq2 RFU for all constitutively active GPCR-Ga strains between $\mathrm{pH} 7$ and 5 . Switch-like Boolean responders to high and low pH are colored cyan and purple, respectively. (D-E) New proton sensors activated by low (D) and high (E) pH. (F) pH titrations of new proton-sensing GPCRs in the context of $\mathrm{pH}$ changes associated with select physiological processes and pathologies. (G) An illustration using ADORA2A proton sensing and $\mathrm{H}^{+}$gating of DRD4 agonism to show how $\mathrm{pH}$ effects on GPCRs can be described by a Boolean language expressed in binary code. (A-C) Vertical dashed lines 
correspond to $\log _{2} \mathrm{FC}=0.5$ and horizontal dashed lines separate examples that signaled more $\left(\log _{2} \mathrm{FC} \geq 0.5\right)$ at $\mathrm{pH} 7$ (blue/cyan bars), similarly $\left(\log _{2} \mathrm{FC}\right.$ between \pm 0.5$)$ between both $\mathrm{pH}$ values (gray bars), or more $\left(\log _{2} \mathrm{FC} \leq 0.5\right)$ at $\mathrm{pH} 5$ (red/purple bars). Filled bars correspond to strains of the known acid sensors (GPR4, GPR65, and GPR68). (A-F) Error bars represent SD of n=4 experimental replicates. 